\title{
Coevolution of cooperation and language
}

\author{
Mohammad Salahshour $\oplus^{*}$ \\ Max Planck Institute for Mathematics in the Sciences, Inselstrasse 22, D 04103 Leipzig, Germany
}

(Received 18 June 2020; accepted 29 September 2020; published 19 October 2020)

\begin{abstract}
As a cooperative act decreases an individual's fitness for others to benefit, it is expected to be selected against by natural selection. That, how contrary to this naive expectation cooperation has evolved, is a fundamental problem in evolutionary biology and social sciences. Here, by introducing a mathematical model, we show that coevolution of cooperation and language can provide an avenue through which both cooperation and language evolve. In this model, individuals in a population play a prisoner's dilemma game and at the same time try to communicate a set of representations by producing signals. For this purpose, individuals try to build a common language, which is composed of a set of signal-representation associations. Individuals decide in language learning based on their payoff from the prisoner's dilemma game and decide about their strategy in the prisoner's dilemma game based on their success in conveying symbolic information. The model shows cooperators are able to build a common language and protect it against defectors' attempt to decode it. The language channels the benefit of cooperation toward cooperators, and defectors, being banished from the language, are unable to exploit cooperators, and are doomed to extinction.
\end{abstract}

DOI: 10.1103/PhysRevE.102.042409

\section{INTRODUCTION}

The evolution of cooperation poses a fundamental challenge in evolutionary biology: If individuals are to be selected based on their fitness, then any altruistic trait that reduces an individual's fitness for the sake of others is to be selected against [1,2]. Nevertheless, a high level of cooperation is observed in biological and social world [3-5]. As a result of many efforts devoted to resolve this apparent paradox, some mechanisms are identified by which cooperation can evolve in a population of self-interested agents subject to natural selection [6,7]. Kin selection can promote cooperation among relatives [2]. If individuals live in relatively closed groups, then competition between groups can lead to a situation in which groups composed of more cooperative individuals perform better. This promotes cooperation via group (multilevel) selection [8,9]. In repeated interactions between individuals, cooperation can evolve by direct reciprocity [10]. When interactions are not repeated, indirect reciprocity, according to which cooperation increases the chance for the individual to receive benefit, can promote cooperation [11-13]. Spatial selection and network reciprocity can promote cooperation in structured populations by increasing associativity between cooperators $[14,15]$. Tag-based mechanisms promote cooperation through a similar effect by increasing the associativity

\footnotetext{
*mohammad.salahshour@mis.mpg.de; salahshour.mohammad@gmail.com
}

Published by the American Physical Society under the terms of the Creative Commons Attribution 4.0 International license. Further distribution of this work must maintain attribution to the author(s) and the published article's title, journal citation, and DOI. Open access publication funded by the Max Planck Society. between cooperators [16,17]. Finally, voluntary participation, such that individuals have the option to opt out of the game and resort to a safe income, can promote cooperation as well $[18,19]$. Punishment is shown to promote cooperation in public goods games in human experiments [20]. Although, due to second-order free riding on punishers, it seems to be unable to promote cooperation by its own, but it is shown to increase cooperation level if supplemented by another mechanism to avoid free riding [21-24]. In addition, reward is shown to be able to promote cooperation $[25,26]$. Recently, it is shown that strategic signaling can provide a new road to the evolution of cooperation, which seems to be able to explain the evolution and maintenance of costly signals as well [27].

It is argued that language can be another means by which high levels of cooperation has evolved in human populations $[6,28]$. Even more, it is suggested that the positive role of language in the evolution of cooperation can be among the factors which have given rise to the evolution of language [29,30]. However, a mathematical model which shows how language and cooperation can co-evolve is still lacking. Here, we provide this missing piece of the puzzle. To this goal, we consider a model in which individuals in a population are paired to play a collective action game [prisoner's dilemma (PD) game] and at the same time, try to communicate a set of representations by producing signals. For this purpose individuals try to learn a set of associations between signals and representations. That, from whom to learn their associations, is determined by their payoff from the prisoner's dilemma game. In the same way, individuals decide about their strategy in the prisoner's dilemma game based on their success in communication with their coplayer. The model shows cooperators can cooperatively build and protect a protolanguage against defectors' attempts to decode their language. The language allows cooperators to identify each 
other based on their success in conveying symbolic information. In this way, language channels the benefit of cooperation towards cooperators. Cooperation, is the key to the common language. Refraining from cooperation, expels defectors from the language. Being banished from the common language, defectors are unable to exploit cooperators and are doomed to extinction.

\section{THE MODEL}

We consider a population of individuals who can engage in symbolic communication and at the same time play a cooperative task. We model the cooperative task by a PD game. This game is extensively used in the studies on the evolution of cooperation [1,6]. In this game, each individual has two strategies: cooperation and defection. If both individuals cooperate, both receive a payoff $R$. If one defects while the other cooperates, the defector receives a payoff $T$, and the cooperator receives a payoff $S$. If both defect, then both get a payoff $P$. The payoffs are such that, $S<P<R<T$ and $T<2 R$. With these conditions, this game constitutes a social dilemma: Cooperation offers the highest total payoff while defection is the most rational strategy. To begin with, we set the payoff values equal to $S=0, R=1, P=0.1$, and $T=1.5$. Later we study how the model behaves for other values.

We assume individuals can also engage in symbolic communication by producing signals to signify a set of representations or meanings that they have [31-38]. For this purpose they have access to $n$ signals to signify $n$ representations. In addition, they are equipped with two communication channels, for production and comprehension of signals. Production channel, $P_{\alpha}(\sigma \mid r)$ gives the probability that signal $\sigma$ is produced for representation $r$, and comprehension channel $Q_{\alpha}(r \mid \sigma)$ gives the probability that representation $r$ is comprehended when receiving signal $\sigma$. Here, the index $\alpha$ refers to an individual $\alpha$. Individuals learn their languages, i.e., $P$ and $Q$, by forming associations between representations and signals from their experience, such that $P_{\alpha}(\sigma \mid r)=\frac{A_{\alpha}(\sigma, r)}{\sum_{\sigma} A_{\alpha}(\sigma, r)}$ and $Q_{\alpha}(r \mid \sigma)=\frac{A_{\alpha}(\sigma, r)}{\sum_{r} A_{\alpha}(\sigma, r)}$. Here, $A_{\alpha}(\sigma, r)$ is the association between signal $\sigma$ and representation $r$, for individual $\alpha$.

The cooperative game and the coordination games are coupled. To model this, we assume individuals first play a language game in which one is the speaker and the other is the hearer, and try to communicate over $l$ randomly chosen representations. That is, in each of the $l$ communication attempts, the speaker produces a signal $\sigma$ for a randomly chosen representation $r_{s}$ and the hearer guesses a representation $r_{h}$ based on the signal. Individuals can be defectors or conditional cooperators. Defectors always defect. Conditional cooperators, however, cooperate if $l_{t}$ out of $l$ communication attempts are successful (i.e., $r_{s}=r_{h}$ for $l_{t}$ times out of $l$ attempts) and defect otherwise. $l$ is a measure of how much individuals have the opportunity to engage in symbolic communication, and $l_{t}$ is a measure of how stringent a conditional cooperator is to cooperate. After the PD game, players coordinate if the opponent cooperates (i.e., increase the association between the signals and representations which led to a successful communication with an amount $d$ ). However, if they cooperate and the opponent defects, then they anticoordinate (i.e., decrease the association between the signals and representation which led to a successful communication by an amount $d$ ).

We consider two different population structures, a wellmixed population, and a structured population. In the wellmixed population, at each time step, individuals are paired at random to play the games. In the case of the structured population, the individuals are set on a network and at each time step play the games with all their neighbors. As the population network, we consider a first-nearest-neighbor square lattice with periodic boundaries. The motivation behind this choice resides in the fact that lattices are a good approximation for a spatial structure, where many evolutionary processes take place. Besides, the simplicity and genericity of lattices allows us to make sure the observed behavior of the model is rather generic and does not result from ad hoc aspects of the network structure. For these reasons, latices are extensively used in many of the studies in evolutionary games $[14,15,19,21,23]$.

Individuals gather payoff according to the outcome of the prisoner's dilemma game. After playing the games, a selection occurs in which an individual is selected with a probability proportional to its payoff and produces an offspring. The offspring replaces a randomly chosen individual. With probability $1-v$, the offspring inherits the strategy of its parent and with probability $v$, a mutation occurs in which case the strategy of the offspring is randomly set to be either $C$ or $D$. The association matrix of the offspring $o$ is given by $A_{o}=$ $(1-\eta) A_{p}+\eta\left[\frac{1}{n^{2}}\right]$. Here, $\left[\frac{1}{n^{2}}\right]$ is a matrix all whose entries are equal to $\frac{1}{n^{2}}$, and $A_{p}$ stands for parent's association matrix. $\eta$ can be seen as the amount of noise in parental learning, and $1-\eta$ is a measure of the amount of parental learning: with $\eta=0$ the offspring learns the language of its parent with certainty, and with $\eta=1$, it starts with a uniformly random language and has to start from scratch to learn a language.

\section{RESULTS}

In Fig. 1, the population fraction of conditional cooperators, $m$, for a population of size $N=100$ as a function of time is plotted (solid blue line). In Fig. 1(a), the case of a wellmixed population is considered and in Fig. 1(b), a population residing on the lattice is considered. Here, and in the following simulations (unless otherwise stated), we set: $\eta=0.1, l=10$, $l_{t}=2, n=50, d=0.1, v=0.01$, and individuals start with random language and strategies. As can be seen in Fig. 1, a large fraction of conditional cooperators is maintained in the population. This results from the fact that cooperators are able to form a common language and preserve their language from defectors' attempts to decode it. As a measure of individuals' success to communicate symbolic information, we consider the population consistency of language defined as [31] $P C=\frac{1}{2 N n} \sum_{\alpha, \beta=1}^{N} \sum_{\sigma, r=1}^{n}\left[Q^{\alpha}(r \mid \sigma) P^{\beta}(\sigma \mid r)+\right.$ $\left.Q^{\beta}(r \mid \sigma) P^{\alpha}(\sigma \mid r)\right]$, and plot this in Fig. 1(a) (red dashed line). As individuals start off with random languages, $P C$ takes a small value in the beginning of the simulation. As time unfolds, individuals begin to form a common language and $P C$ increases. Interestingly, $m$ and $P C$ evolve in a correlated fashion. When $P C$ is large, cooperators exchange symbolic information more easily, and can recognize each other through linguistic interactions. This allows them to 

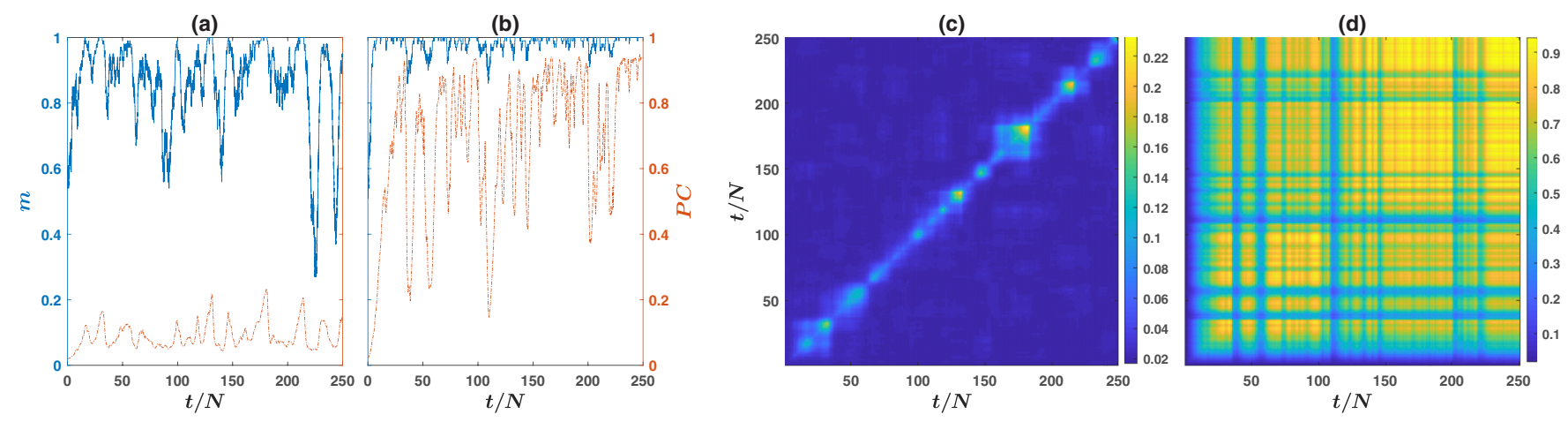

FIG. 1. Time dependence of the model. (a), (b) The population fraction of conditional cooperators $m$ (blue solid line), and the population consistency of the language $P C$ (red dashed line), a measure of how well individuals understand each other. In panel (a) a well-mixed population is considered and in panel (b) the population resides on a first-nearest-neighbor square lattice with periodic boundaries. Cooperation, and a common language evolve in a correlated fashion indicated by bursts where both take a high value, and periods when both drop. (c), (d) Color plot of the time-dependent population consistency, a measure of how well individuals at different generations understand each other, in a well-mixed population (c) and for a population on a lattice (d). While in a well-mixed population, population consistency of language remains small, for a structured population, language reaches a high level of population consistency and historical similarity.

reach a high payoff by selectively cooperating with each other. This in turn increases the cooperation level, $m$. Occasionally, defectors are able to decode the language. This increases the payoff of defectors and thus reduces $m$. In such times, cooperators, being defected by defectors who understand their language, attempt to change their language to keep defectors away. This leads to a sharp decline in the value of $P C$, which signals a period of language change in the population, until cooperators are able to reestablish another language immune from defectors. Fluctuation between these two states of high and low cooperation and symbolic information exchange level, is a fundamental characteristic of the model.

The simulation on the lattice, presented in Fig. 1(b) shows a similar phenomenology as in the case of a well-mixed population. The main difference however, is that network structure facilitates language formation and thus, cooperation. This can be seen by noting that both $m$ and $P C$ take significantly larger values on a network compared to a well-mixed population. The reason is that, on a network with fixed interactions, neighboring conditional cooperators who are willing to cooperatively form a common language, are able to do so without defectors' intrusion. We note that, even though network structure is known to facilitate cooperation with certain update rules [15], under our update rule (random replacement of an individual with the newly born offspring of the winner), cooperation does not emerge without a language. In this regard, our analysis reveals a fundamentally new way in which network structure promotes cooperation by facilitating coordination between cooperators to build a common set of conventions to communicate symbolic information.

In our model, cooperators are under constant pressure to rearrange their language to keep it immune from defectors. This provides an avenue for language change. An interesting question is how language evolves in time and how languages formed at different times are related. To investigate this, we define the time-dependent population consistency as $\quad P C_{t}\left(t_{1}, t_{2}\right)=\frac{1}{2 N n} \sum_{\alpha, \beta=1}^{N} \sum_{\sigma, r=1}^{n}\left[Q_{t_{2}}^{\alpha}(r \mid \sigma) P_{t_{1}}^{\beta}(\sigma \mid r)+\right.$ $\left.Q_{t_{1}}^{\beta}(r \mid \sigma) P_{t_{2}}^{\alpha}(\sigma \mid r)\right]$. This is a measure of how individuals living in generations $t_{1}$ and $t_{2}$ understand each other. Generation time is defined as the simulation time divided by the population size $N$. We color plot $P C_{t}$ as a function of generation time in Fig. 1(c), in the case of a well-mixed population, and in Fig. 1(d) in the case that individuals reside on a fixed network of interactions [this is based on the same simulation presented in Figs. 1(a) and 1(b)]. As can be seen, language constantly changes. At some times, a language emerges (this corresponds to a bright color on the diagonal). However, after a while, defectors manage to decode the language, and consequently, the language collapses. In a well-mixed population, usually, the newly emerged language has no similarity with its predecessors. But in some instances [such as the language emerged between generations 150 and 200 in Fig. 1(c)], a future language results from an old one by small rearrangements. In contrast, this is most often the case on a network, where cooperators are able to keep their language immune by small changes. Consequently, the core of the language is preserved over time and many generations are able to significantly understand each other. Comparison between well-mixed and networked populations suggest that, a network structure is highly facilitating for a language to reach a high level of population consistency and historical similarity. An important question is how our results depend on the parameter values. To address this question, in Fig. 2 we look at the parameter dependence of our results. Here, time averages of $m$ and $P C$, as a function of model parameters for both population structures are plotted (squares indicate simulation results for population on a lattice, and circles indicate results for a well-mixed population). We begin by investigating the dependence on $l_{t}$ in Fig. 2(a). As can be seen, for a given strength of communication $(l=10)$ an optimal $l_{t}$ exists: for smaller $l_{t} \mathrm{~s}$ individuals are too liberal and defectors can easily deceive cooperators. For larger $l_{t} \mathrm{~s}$, cooperators are too chauvinist such that the formation of a common language becomes increasingly difficult starting from a random language (as the formation of a common language necessitates individuals occasionally cooperate by chance to be able to build a common language, the chances of which decreases with increasing $l_{t}$ ). 

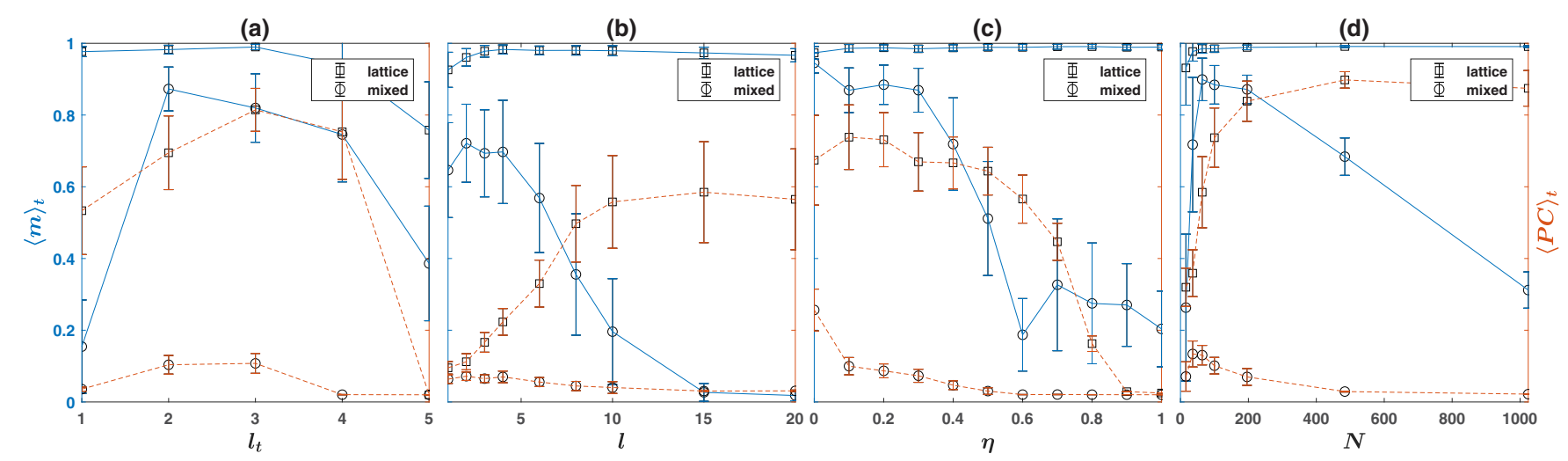

FIG. 2. Dependence on the model parameters. The time average cooperation level, $\langle m\rangle_{t}$ (blue), and the population consistency of the language (red), $\langle P C\rangle_{t}$, as a function of model parameters, for two different population structures. Cooperation evolves in a broad range of parameter values. The level of cooperation (blue solid line) and population consistency of language (red dashed line), are much higher for a structured population (squares) compared to a well-mixed population (circles). $l_{t}$ is the number of correct communications necessary for a conditional cooperator to cooperate, $l$ is the number of communications before a game, $\eta$ the amount of noise in parental learning, and $N$ the population size. The network used here is a first-nearest-neighbor lattice with periodic boundaries. The simulations are performed for $10^{5}$ time steps and time averages and standard deviations (error bars) are calculated after discarding the first $10^{4}$ time steps.

The dependence on the strength of communication $l$, is investigated in Fig. 2(b). Here, $l_{t}$ is set to 1 , and $m$ and $P C$ as a function of $l$ are plotted. As observed in the figure, even one communication instance $(l=1)$ can be enough for the emergence of cooperation. Furthermore, as $l$ increases, individuals have more chances to coordinate and both $\langle P C\rangle_{t}$ and $\langle m\rangle_{t}$ increase. However, for larger $l$, the level of cooperation decreases. The reason is that with fixed $l_{t}$ the probability that a conditional cooperator cooperates with a defector increases with increasing $l$. Such interactions favor defectors and thus, the level of cooperation decreases. In addition, such interactions cause cooperators to constantly change their language as they are defected frequently. This in turn decreases $P C$.

Dependence on parental learning is investigated in Fig. 2(c). In a well-mixed population, the level of cooperation decreases with decreasing parental learning (i.e., by increasing the noise $\eta$ in parental learning). However, the situation is different on a network: For a very small amount of noise in parental learning, the level of cooperation slightly increases by increasing the noise in parental learning. The reason is that, a too small amount of noise in parental learning allows the defectors to decode the language by learning from cooperators' offspring in their neighborhood. This cannot happen for larger amount of noise in parental learning, as in this case, offspring language bears less similarity with that of its parent and cannot be exploited by defectors as a door to the language.

The dependence on the population size $N$, is investigated in Fig. 2(d). For too small populations, the same individuals interact too often which gives the defectors a high opportunity to decode cooperator's language. Consequently, the level of cooperation is small for too small population sizes in both cases of the well-mixed and the networked populations. In large populations however, the situation is different for the two population structures. In the networked population, language can maintain cooperation even for very large sizes. In addition, $P C$ increases with population size. The reason is that, by increasing the population size, islands of cooperators can form with no defector in the close vicinity. Such small com- munities of cooperators provide safe places for cooperators to coordinate their language and play an important role in the formation and maintenance of cooperator's language. On the contrary, for a mixed population, for a too large population size, it becomes increasingly difficult for the cooperators to coordinate on a common language and consequently the level of cooperation and $P C$ drop.

Interestingly, the model shows there exists an optimal population size which facilitates the evolution of language and maximizes the level of cooperation: For too small population sizes, it is difficult to keep defectors away, and for two large population sizes, it becomes difficult for conditional cooperators to coordinate on a common language in the face of defectors. In the Appendix $C$ we confirm that the existence of an optimal population size holds for a broad range of parameter values. The optimal population size however, can slightly depend on the parameter values. It seems tempting to see if this result can bring any insight into the evolution of language and cooperation in early human groups. It is argued that the typical size of early human groups was of the order of 100 individuals [39]. For a rather broad range of parameter values in our model, this size falls in the regime where a significant level of cooperation and language consistency can evolve in a well-mixed population. This can be argued to suggest that a protolanguage could have been an effective mechanism to promote cooperation in such group sizes, and thus, a strong selection pressure for the evolution of language could have been at work due to its role in promoting cooperation.

In the Appendices, we further investigate the dependence of the model behavior on other parameters of the model (Appendix B). In addition, we show that cooperation can evolve in a structured population of unconditional cooperators and defectors as well (Appendix E). In the case of a well-mixed population of unconditional cooperators and defectors, cooperation can evolve with a weak prisoner's dilemma $(S=P)$ [40]. We also consider a birth-death update rule, in which the offspring replaces one of the neighbors of its parent (Appendix D). Without a language, competition 
between cooperators over scarce sites prevents cooperation to evolve for such an update rule [15]. However, as shown in the Appendix D, a high level of cooperation evolves when individuals have access to a language to exchange symbolic information.

\section{DISCUSSION}

While it had been suggested that language may have played an important role in the evolution of cooperation in human groups [28], and furthermore, language's positive role in the evolution of cooperation may have contributed to the evolutionary pressure to develop language [29,30], a mathematical model that shows how this could happen was missing. Here, we have tried to proved a simple mathematical model to cast light on this subject. To this goal, we have regarded language as a set of associations between a set of representations and signals which individuals use to convey symbolic information. This can be seen as a simplified model of language [41,42], or a protolanguage at work at the early stages of the evolution of human language [31-35]. More generally, a set of associations between representations and signals, as considered here, can be seen as a model of biological communication in many species in which individuals communicate by signal exchange [37,38]. In this regards, our model shows how cooperative construction of a shared set of linguistic conventions to communicate symbolic information can provide an avenue for the emergence of cooperation in different communicating species with primitive cognitive abilities.

In our model, language is acquired by cooperative coordination among cooperators. More precisely, a common language is acquired by cooperators' attempt to make their language more similar to fellow cooperators and differentiate their language from that of defectors. This, arguably, can result from individuals' willingness to identify and cooperate with like-minded cooperators [43,44], and as our analysis shows, leads to a situation where a cooperative act serves as a token which is the key for the individual to enter the common language. Defectors, not having the key, are banished from the language and are unable to exploit cooperators. Cooperators' attempt to build and protect a cooperative language with likeminded cooperators, in the face of defectors' attempt to find the door to the language, provides an avenue for language changes and leads to correlated fluctuations in the population consistency of the language and the level of cooperation. These fluctuations can be suppressed by a network structure, which facilitates building a common language and promotes cooperation. In this regards, our analysis reveals yet another way in which assortativity resulted from a network structure can facilitate the evolution of cooperation, by providing cooperators the opportunity to build a common set of linguistic conventions. However, in a well-mixed population, defectors perform better in decoding the common language and exploiting cooperators. This decreases the level of population consistency and cooperation and leads to fluctuations in which different languages are formed, but before going to fixation are decoded by defectors and go to extinction.

Our model provides insights into the evolution of language as well. Computational models aiming at modeling the evolution of language, have shown that how in a population of agents in which communication is mutually beneficial, language can evolve $[31,32,41,42]$. We have extended these researches by showing a symbolic communication system can evolve, even if communication endows no direct benefit. In this regard, language can provide a collective identity, which benefits the individuals who contribute to building it by costly cooperation. This is done by providing a mean to avoid exploitation by those who do not contribute in forming the shared language. In this way, language increases the overall group fitness by promoting cooperation.

\section{ACKNOWLEDGMENT}

The author acknowledges funding from Alexander von Humboldt Foundation in the framework of the Sofja Kovalevskaja Award endowed by the German Federal Ministry of Education and Research.

\section{APPENDIX A: OVERVIEW OF THE MODEL AND SIMULATIONS}

In this Appendix, we bring an overview of the model, and explain the simulations.

We consider a population of $N$ individuals, who are able to communicate by production and comprehension of signals, and play a prisoner's dilemma (PD) game. Individuals collect payoff according to the outcome of the prisoner's dilemma game and are subject to selection based on their payoff. In the following, each part of the model is explained in turn.

\section{The model of language}

Individuals have access to a set of representations or meanings $\mathbf{r}=\left\{r_{1}, . ., r_{n}\right\}$ and are able to produce and comprehend $n$ signals $\Sigma=\left\{\sigma_{1}, \ldots, \sigma_{n}\right\}$, which they use to communicate their representations. For this purpose, each individual $\alpha$ is equipped with two communication channels. Production matrix $P_{\alpha}(\sigma \mid r)$ gives the probability that signal $\sigma$ is produced for representation $r$. In the same way, comprehension matrix $Q_{\alpha}(r \mid \sigma)$ gives the probability that representation $r$ is comprehended when receiving signal $\sigma$. Individuals can form associations between signals and representations. Association between signal $\sigma$ and representation $r$ is denoted by $A_{\alpha}(\sigma, r)$. Individuals form their production and comprehension matrices based on their associations, by setting $P_{\alpha}(\sigma \mid r)=$ $\frac{A_{\alpha}(\sigma, r)}{\sum_{\sigma} A_{\alpha}(\sigma, r)}$ and $Q_{\alpha}(r \mid \sigma)=\frac{A_{\alpha}(\sigma, r)}{\sum_{r} A_{\alpha}(\sigma, r)}$. Individuals change their associations based on the payoffs they receive in the prisoner's dilemma game as described below.

\section{Dynamics of the games}

When two individuals are paired to interact, they first try to communicate, and then play a PD game. Their strategy in the PD is determined based on the result of the communication. To communicate, one of the individuals, chosen at random, becomes the speaker and the other one becomes the hearer. The speaker produces a signal $\sigma$ for a randomly chosen representation using her production matrix, and the hearer guesses a representation using her comprehension matrix. Communication is done for $l$ rounds, in each round over a randomly chosen representation. After the $l$ communication 
attempts, the individuals play a prisoner's dilemma. Defectors always defect. Conditional cooperators, however, cooperate if $l_{t}$ out of $l$ communication attempts is successful, and defect otherwise. After the PD game, individuals receive payoff and update their associations based on their payoff. Individuals always coordinate with cooperators, i.e., an individual coordinates if its payoff is larger than or equal to $R$. However, an individual anticoordinates if she cooperates while the opponent defects. That is, when it receives the minimum payoff $S$. When coordinating, the individual increases her association between the subset of the realized signal-representations which led to a correct communication, by an amount $d$. When anticoordinating, the individual decreases her associations for the subset of signal-representations which led to a correct communication, by an amount $d$. If the association between a signal and representation becomes negative, it is set to a small number, $10^{-60}$. The association matrix is kept normalized in each time step such that the sum of all its elements is equal to 1 , and the comprehension and production matrices are calculated based on the association matrix in each time step.

\section{The evolutionary model}

Two different population structures are considered. In the case of a well-mixed population, at each time step individuals are paired at random to play the games. In the case of a population residing on a network (a first-nearest-neighbor square lattice with periodic boundaries), at each time step, each individual plays the games with all its neighbors. Individuals gather payoff based on the $\mathrm{PD}$ game. In each time step, an individual is selected with a probability proportional to its payoff and produces an offspring. The offspring inherits the strategy of its parent and replaces a randomly chosen individual in the population. However, with probability $v$ a mutation can occur in which case the offspring's strategy is set randomly to be either $C$ (conditional cooperation) or $D$ (defection). The association matrix of the offspring is determined according to $A_{o}=(1-\eta) A_{p}+\eta\left[\frac{1}{n^{2}}\right]$. Here, $\left[\frac{1}{n^{2}}\right]$ is a matrix all whose entries are $\frac{1}{n^{2}}$, and $A_{p}$ stands for parent's association matrix. $\eta$ can be seen as the amount of noise in parental learning, and $1-\eta$ can be seen as a measure of the amount of parental learning: with $\eta=0$ the offspring learns the language of its parent with certainty, and with $\eta=1$, it starts with a uniformly random language and has to start from scratch to learn a language.

\section{The simulations}

The base parameter values used in the simulations are $N=100, \eta=0.1, l=10, l_{t}=2, n=50, d=0.1, v=0.01$, and the base payoff values are $S=0, R=1, P=0.1$, and $T=1.5$. Individuals start with random language and strategies. Simulations are performed for $t=100000$ time steps. For investigating the parameter dependence in Fig. 2, a time average is taken after discarding the first $t=10000$ steps. Error bars reported in the figures are the standard deviation of the corresponding quantity calculated based on this sample. In each simulation in this figure one of the parameters is changed, keeping the rest constant and equal to the base value (unless otherwise stated).

\section{APPENDIX B: DEPENDENCE ON THE MODEL PARAMETERS}

We have investigated the dependence of the cooperation level on the extent of communication $l$, the number of correct communications necessary for a conditional cooperator to cooperate $l_{t}$, the amount of noise in parental learning $\eta$, and the population size $N$, in the main text. Here, we investigate the dependence of the cooperation level on other parameters of the model.

The dependence on the mutation rate in strategies, $v$, is investigated in Fig. 3(a). Here, we see that by increasing $v$ the level of cooperation and $P C$ decrease. The reason is that with a large mutation rate, a cooperator's offspring turns defector too often. Assuming noise in parental learning is small, which is the case here $(\eta=0.1)$, such defectors have a high knowledge of cooperators' language, and thus, can significantly exploit them.

The dependence on the number of representation, $n$, is investigated in Fig. 3(b). With $l=10$ and $l_{t}=2$, which is used here, for too small $n$ cooperation does not evolve. The reason is that the probability that two individuals with random languages have a correct communication increases with decreasing $n$. Consequently, with a small value of $n$, individuals need to set a higher level of $l_{t}$ as a criteria to cooperate. However, for large $n$ this probability decreases and building a common language becomes increasingly difficult starting from a population with random languages. This is why $P C$ decreases for large $n$. In such cases, individuals need to set a lower value of $l_{t}$ to be able to form a language.

The dependence on learning rate, $d$, is investigated in Fig. 3(c). Here, we can see that the level of cooperation increases with increasing the learning rate. However, for very large learning rates $P C$ decreases, as such a fast response can lead to too large changes in the language of an individual in response to an instance of communication.

Finally, the dependence on the payoffs of the game are investigated in Fig. 3(d) for the temptation and Fig. 3(e) for the punishment. While the level of cooperation slightly decreases with increasing the temptation and the punishment, it remains large in the whole range of the prisoner's dilemma game. This results from the fact that language offers a strong way for the cooperators to selectively cooperate with their peer cooperators.

\section{APPENDIX C: EXISTENCE OF AN OPTIMAL POPULATION SIZE FOR THE EVOLUTION OF COOPERATION IN A MIXED POPULATION}

In this section, we show that, in a mixed population, for a broad range of the parameter values, an optimal population size exists which optimizes the cooperation level. To do so, we run simulations for different population sizes to derive the time average cooperation level as a function of the population size. The results are presented in Fig. 4. Here, using the base parameter values, in each panel one of the parameters is changed (as specified in the figure), and the time average cooperation level is calculated. As can be seen, an optimal population size exists for a broad range of parameter values. While the optimal population size shows small sensitivity to 
(a)

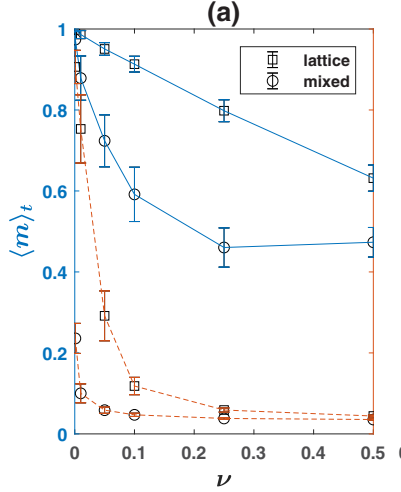

(b)

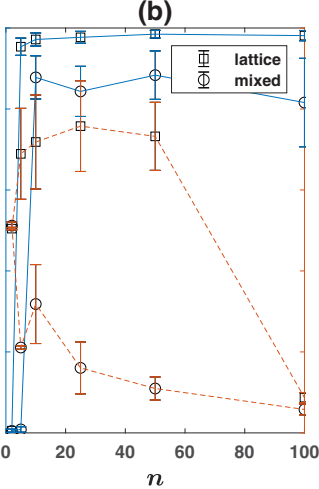

(c)

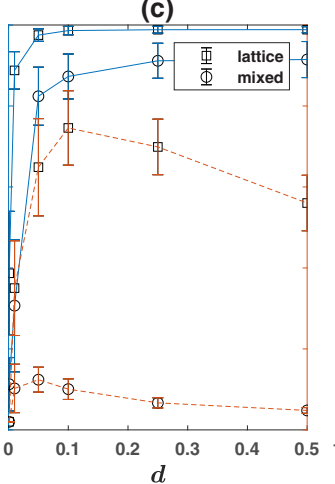

(d)

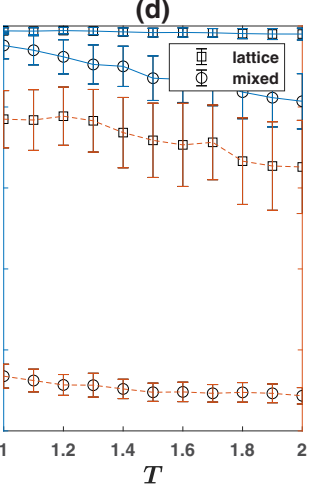

(e)

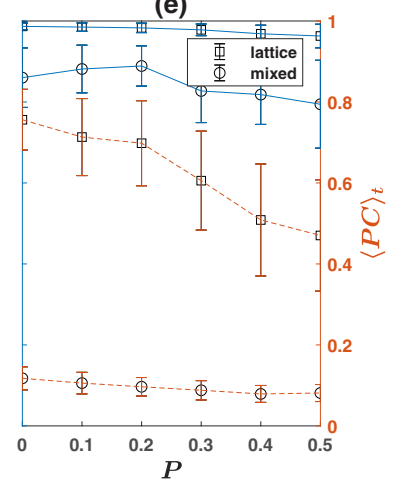

FIG. 3. Dependence on the model parameters. The time average cooperation level, $\langle m\rangle_{t}$ (blue), and the population consistency of the language (red), $\langle P C\rangle_{t}$, as a function of the model parameters, for two different population structures. Cooperation evolves in a broad range of parameter values. The level of cooperation (blue solid line) and population consistency of language (red dashed line), are much higher for a structured population (squares) compared to a well mixed population (circles). $v$ is the mutation rate in strategies, $n$ is the number of representations (equal to the number of signals), $d$ is the learning rate, $T$ the temptation, and $P$ the punishment. The network used here is a first-nearest-neighbor square lattice with periodic boundaries. The simulations are performed for $10^{5}$ time steps and time averages and standard deviations (error bars) are calculated after discarding the first $10^{4}$ time steps.

the language adjustment rate $d$, noise in parental learning $\eta$, mutation rate in strategy $v$, the number of representations $n$, and the number of communications $l$, a larger dependence on the number of correct communications needed for a conditional cooperator to cooperate, $l_{t}$, is observed. Increasing $l_{t}$ while keeping $l$ fixed, decreases the probability that conditional cooperators cooperate. This gives an advantage to conditional cooperators, as such stringent conditional cooperators are exploited less frequently by defectors, and thus, can effectively build a common language and dominate the population even in larger population sizes. However, for too large $l_{t}$ (for a fixed $l$ ), the probability that conditional (b)

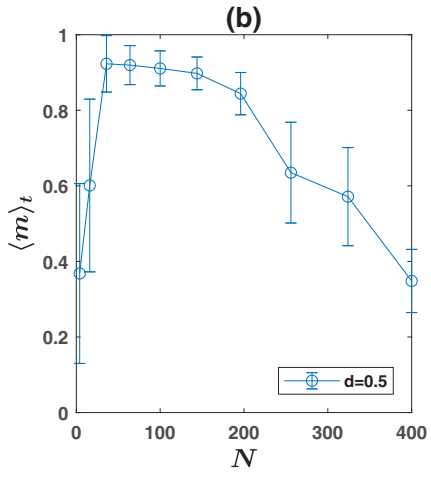

(e)

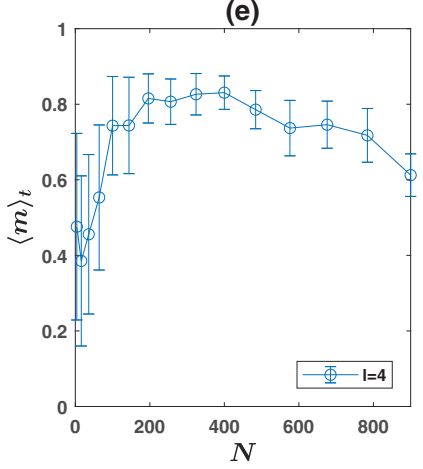

(b)

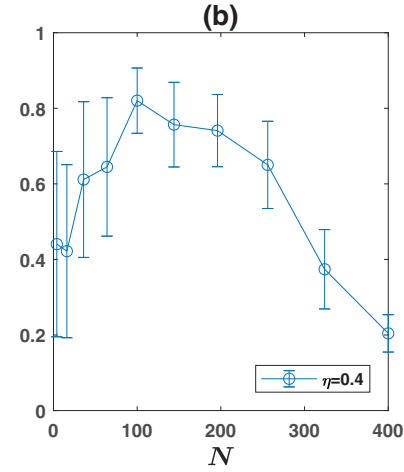

(f)

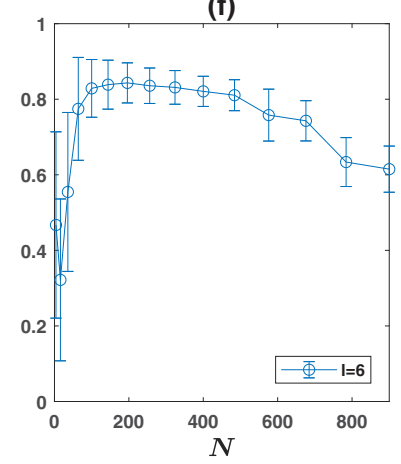

(c)

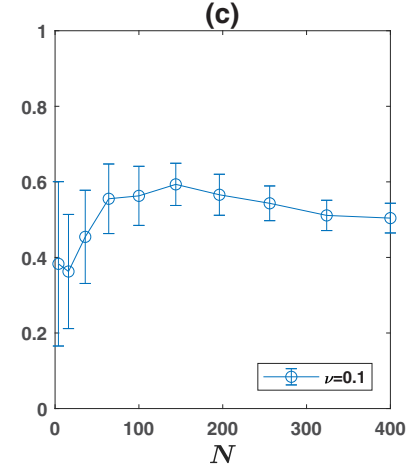

(g)

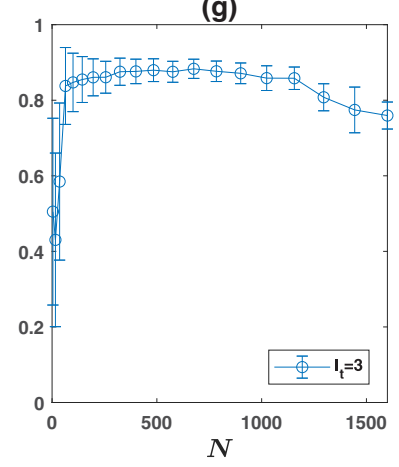

(d)

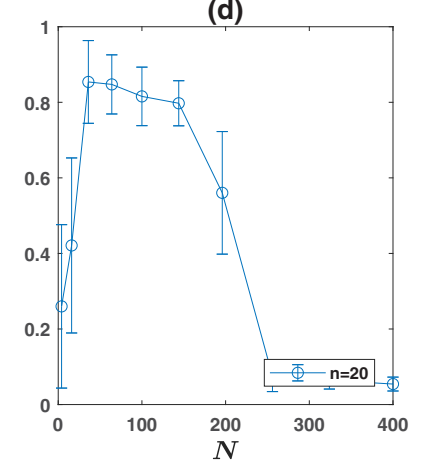

(h)

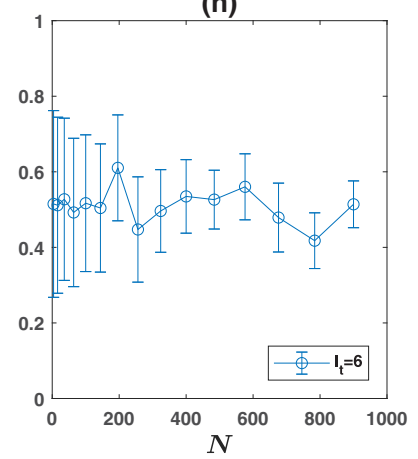

FIG. 4. Existence of an optimal population size for the evolution of cooperation in a mixed population. The average cooperation level is maximized in an intermediate population size for different parameter values. Here, the base parameter values are used. In each panel, one of the parameters is changed as specified in the figure and the time average cooperation level is plotted as a function of population size. In panels (a)-(c), the simulations is run for $T=4 * 10^{5}$ time steps, and in panels (d)-(h) it is run for $10^{6}$ time steps. In all the cases, the average is taken after discarding the first $10^{4}$ time steps. Error bars show standard deviation calculated based on the same sample. 
(a)

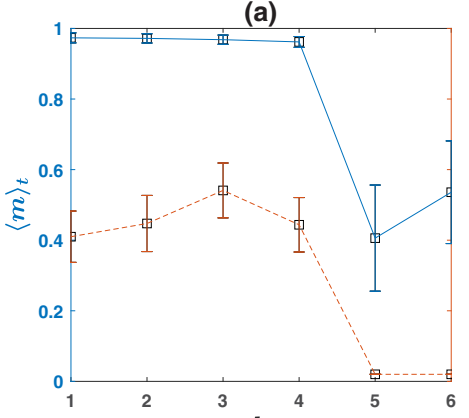

(e)

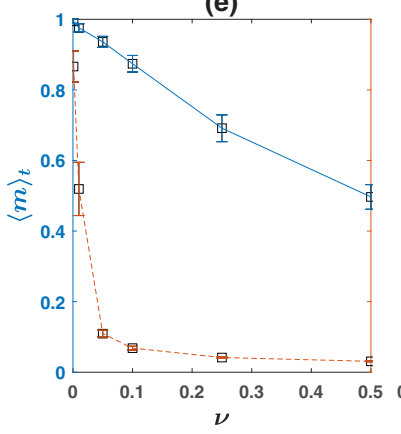

(b)

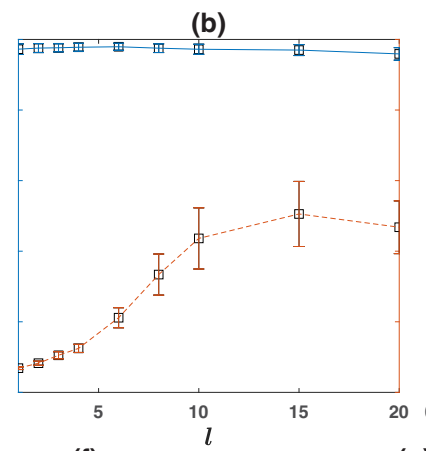

(f)

(g)
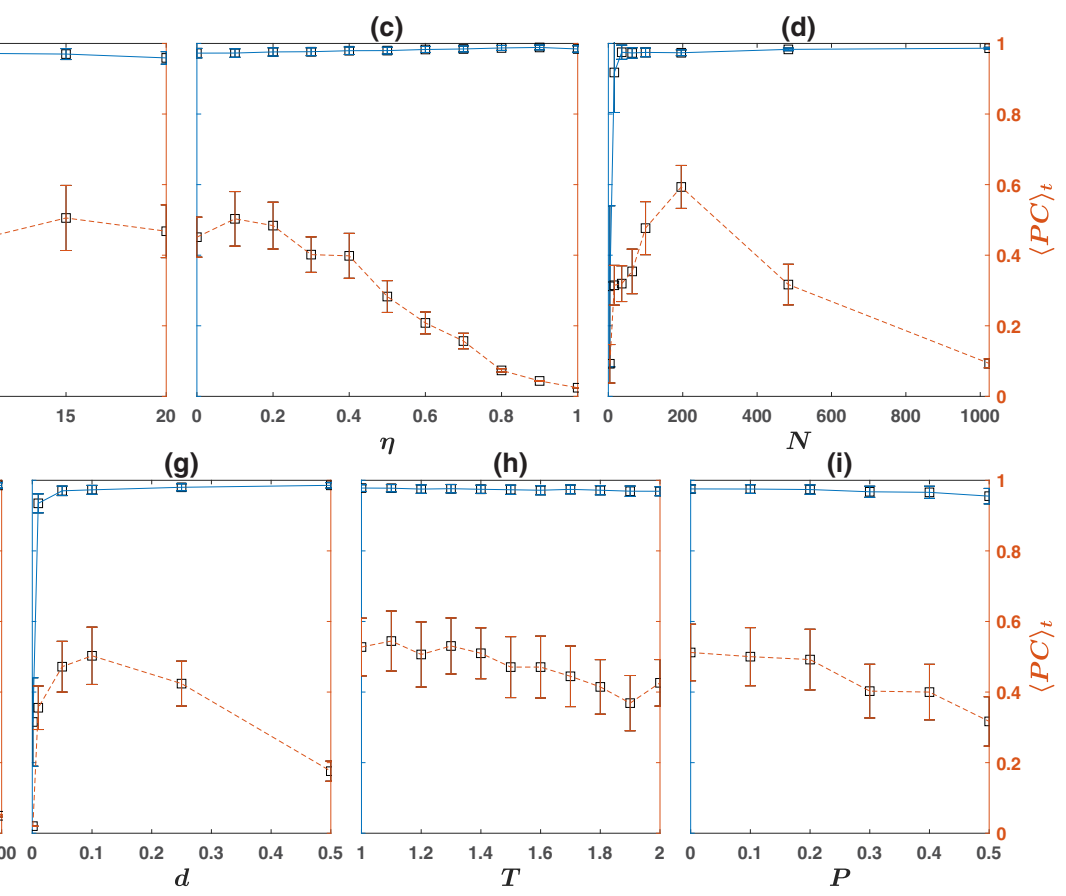

FIG. 5. Dependence on model parameters in the model with birth-death update rule. The time average cooperation level, $\langle m\rangle_{t}$ (blue), and the population consistency of the language (red), $\langle P C\rangle_{t}$, as a function of model parameters, for a structured population. Cooperation and a common language evolve and are maintained in a broad range of parameter values. $l_{t}$ is the number of correct communications necessary for a conditional cooperator to cooperate, $l$ is the number of coordination before a prisoner's dilemma game, $\eta$ is the amount of noise in parental learning, $N$ is the population size, $v$ is the mutation rate in the strategies, $n$ is the number of representations (equal to the number of signals), $d$ is the learning rate, $T$ the temptation, and $P$ the punishment. The simulations are performed for $10^{5}$ time steps and time averages and standard deviations (error bars) are calculated after discarding the first $10^{4}$ time steps.

cooperators successfully communicate and cooperate with each other, significantly decreases. In such a context, conditional cooperators most often defect, receive the same payoff as defectors, and coexist with defectors almost in the same frequency. However, although conditional cooperators are maintained in the population in this regime, a common language does not evolve, due to the lack of positive cooperative interactions among conditional cooperators.

\section{APPENDIX D: BIRTH-DEATH UPDATE RULE ON A NETWORK}

Here, we consider a population residing on a network (a first-nearest-neighbor square lattice with periodic boundaries) with a birth-death update rule. That is, at each time step that an individual is selected for reproduction, its offspring replaces a randomly chosen neighbor of the parent. While, it is known that network structure can promote cooperation, the competition between cooperators over scarce sites reduces the benefits arising from network reciprocity and precludes cooperation with a birth death update rule [15]. However, as we show here, the situation changes when individuals are able to engage in linguistic communication, and cooperation evolves.

In Fig. 5, we investigate the level of cooperation with respect to the model parameters, with a birth-death update rule. As can be seen, a high level of cooperation evolves in the population. The dependence of the cooperation level on the model parameters is similar to the model with random death update rule, and thus we do not elaborate on them here. This shows that the details in the implementation of the dynamics does not affect the overall behavior of the model. Network structure facilitates the evolution of cooperation by facilitating for the neighboring cooperators to collectively build a common language. The language provides a way for cooperators to channel cooperation to fellow cooperators and avoid exploitation by defectors.

\section{APPENDIX E: EVOLUTION OF UNCONDITIONAL COOPERATION IN A STRUCTURED POPULATION}

We consider a second model in which cooperators never defect. Instead, they can decide not to play the prisoner's dilemma game if the communication is not successful. More specifically, in this model, at each time step, individuals play the games with their neighbors on the network. Each pair plays $l$ rounds of a coordination game in which one is speaker and the other hearer. In each round of the coordination game, the speaker produces a signal for a randomly chosen representation $r$, according to its production matrix $P(\sigma \mid r)$. The hearer guesses a representation $r_{h}$ for the received signal according to its comprehension matrix $Q(r \mid \sigma)$. After the $l$ rounds of the coordination game, they have the possibility of playing a prisoner's dilemma game. Defectors always play the PD game and defect. Cooperators however, play the PD only if the fraction of correct communications is equal to, or larger than $l_{t}$. If deciding to play the $\mathrm{PD}$, cooperators always cooperate. It can be argued, intuitively, that the evolution of cooperation with such unconditional cooperators is more difficult 
(a)

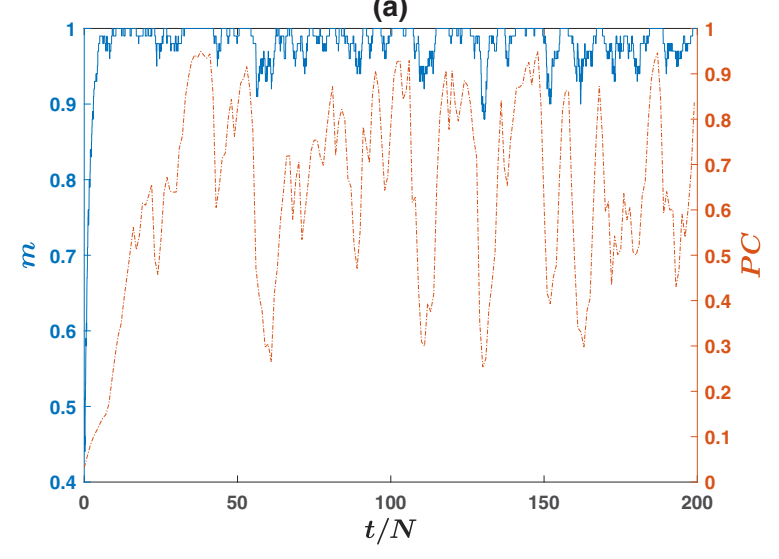

(b)

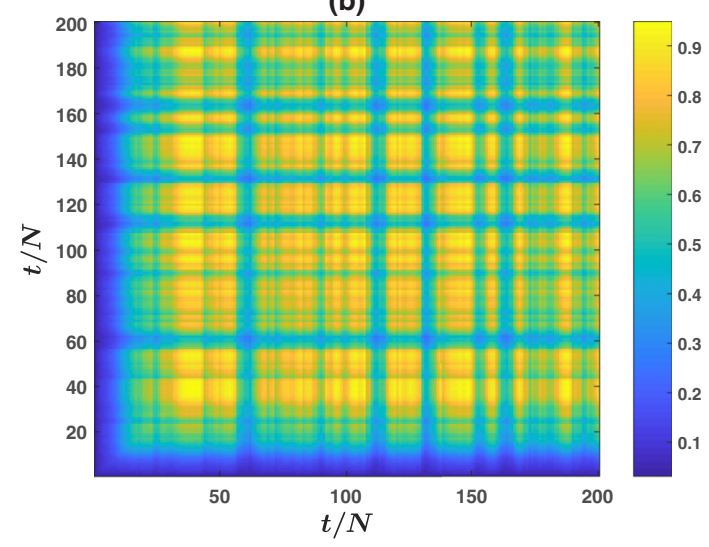

FIG. 6. Time dependence of the model for a population of unconditional cooperators on a first-nearest-neighbor square lattice. (a) The population fraction of cooperators $m$ (blue solid line) and the population consistency of the language $P C$ (red dashed line). Cooperation, and a common language evolve in a correlated fashion indicated by bursts where both take a high value, and periods when both drop. (b) Color plot of the time depended population consistency, a measure of how well individuals at different generations understand each other. Cooperators are under constant pressure to change their language to keep it incomprehensible for the defectors. Languages evolved in different times show historical similarity. Which means the same language is preserved by small modifications to purge the defectors away.

compared to the model with conditional cooperators. The reason is that, defectors, by playing with each other can receive a payoff of $P$ which is larger than the payoff of cooperators when they decide not to play the game (which is set to 0 ). However, as can be seen in Fig. 6(a) cooperation can evolve in this model as well, when the individuals reside on a network. As usual, the network is a first-nearest-neighbor square lattice with periodic boundary condition, and the same parameter values used in the main text is used $(N=100, n=50$, $l=10, l_{t}=2, \eta=0.1, v=0.01 d=0.1, S=0, P=0.1$, $R=1$ and $T=1.5$ ). Here, the fraction of cooperators in the population as a function of time is plotted (solid blue line). The population consistency of the language, defined as $P C=$ $\frac{1}{2 N n} \sum_{\alpha, \beta=1}^{N} \sum_{\sigma, r=1}^{n}\left(Q^{\alpha}(r \mid \sigma) P^{\beta}(\sigma \mid r)+Q^{\beta}(r \mid \sigma) P^{\alpha}(\sigma \mid r)\right)$, is plotted too (red dashed line). As can be seen, cooperators are able to form and protect a common language. Making their strategy conditional on understanding, cooperators can survive and exist in a large fraction. Defectors constantly attempt to understand cooperators' language. At some time instances, they succeed and persuade cooperators to play the PD game. As defectors receive larger payoff compared to cooperators at such times, the fraction of cooperators decreases. Cooperators in turn, being defected, rearrange their language to keep it away from defectors. This in turn reduces the PC and cooperator's ability to understand each other. After a while, cooperators manage to form a new language which is not understandable for defectors. This dynamics is similar to the model with conditional cooperators.

In Fig. 6(b), we look at the language changes over time. To do so, we color plot the time-dependent population consistency of the language, $P C_{t}\left(t_{1}, t_{2}\right)=$ $\frac{1}{2 N n} \sum_{\alpha, \beta=1}^{N} \sum_{\sigma, r=1}^{n}\left[Q_{t_{2}}^{\alpha}(r \mid \sigma) P_{t_{1}}^{\beta}(\sigma \mid r)+Q_{t_{1}}^{\beta}(r \mid \sigma) P_{t_{2}}^{\alpha}(\sigma \mid r)\right]$, as a function of generations (defined as simulation time divided by the population size). As described in the main text, $P C_{t}\left(t_{1}, t_{2}\right)$ is a measure of how well individuals living at times $t_{1}$ and $t_{2}$ understand each other [this is symmetric under the exchange of $t_{1}$ and $t_{2}$, and we have $\left.P C_{t}\left(t_{1}, t_{1}\right)=P C\left(t_{1}\right)\right]$.
In the beginning of the simulation, individuals have started with a random language. Consequently, they are not able to understand each other, neither they can understand individuals at later times. This can be observed by noting the dark color when either $t_{1}$ or $t_{2}$ is small. As time unfolds, a common language starts to form. This happens around generation 10 (where $P C_{t}$ takes a value denoted by green color). However, the formation of the language is not a gradual process and language is subject to constant changes. This results from defectors' attempts to understand the common language, and cooperators' attempts to keep their language safe (incomprehensible to defectors) by modifying it. Consequently, as can be seen in Fig. 6(b) there comes periods where the population consistency of the language drops. However, a characteristic of the languages evolved in networked populations is historical similarity. That is, a language evolved at a time is understandable for other generations [this can be seen in Fig. 6(b) by noting that $P C\left(t_{1}, t_{2}\right)$ for a fixed $t_{1}$ has several peaks (periods with bright color) for different times $t_{2}$ ]. This results from the fact that when a language is partially disposed to defectors, cooperators are able to make it incomprehensible for defectors again by small modifications of the language. As seen in the main text, this is a property of the language change in networked populations. In contrast, in a well-mixed population the historical similarity of languages evolved in different times is significantly lower.

Finally, the parameter dependence of the model with unconditional cooperators is investigated in Fig. 7. Here, the time averages of $m$ and $P C$ are plotted as a function of model parameters. The overall parameter dependence of the model with unconditional cooperators is similar to that with conditional cooperators introduced in the main text. Below, we explain the parameter dependence in detail. The dependence on the number of correct communications necessary for a cooperator to play the PD game $l_{t}$, is investigated in Fig. 7(a). As mentioned before, $l_{t}$ is a measure of how cooperators are 
(a)

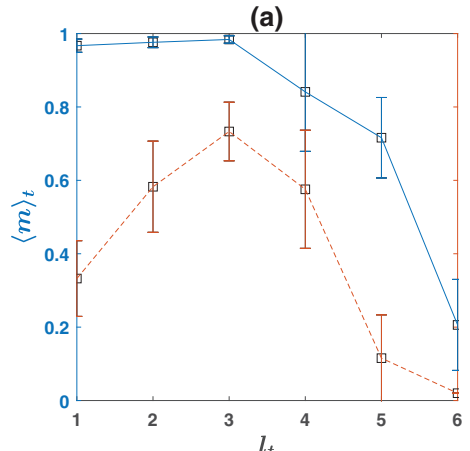

(e)

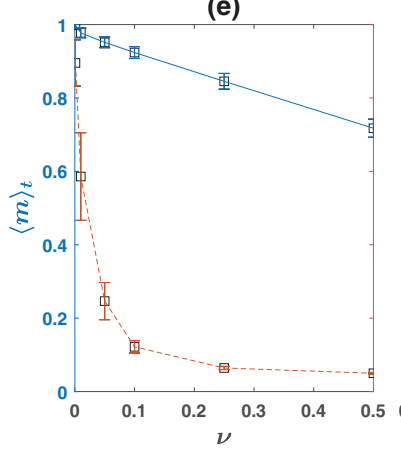

(b)

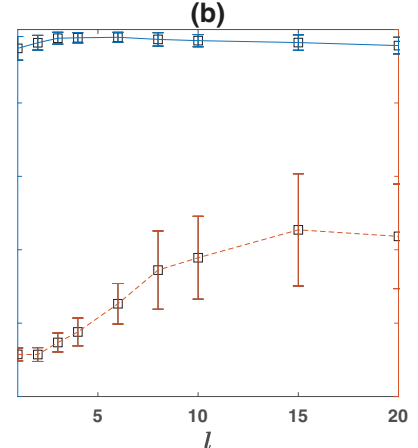

(c)

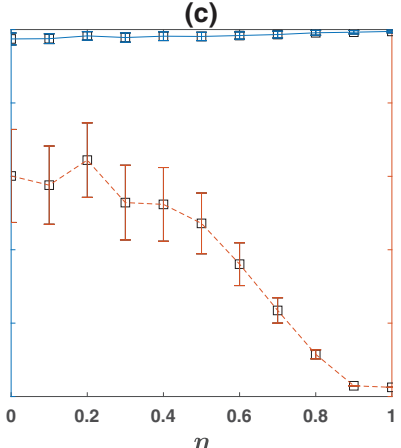

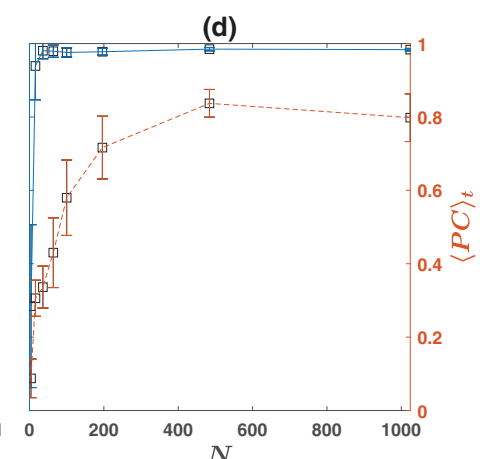

(g)
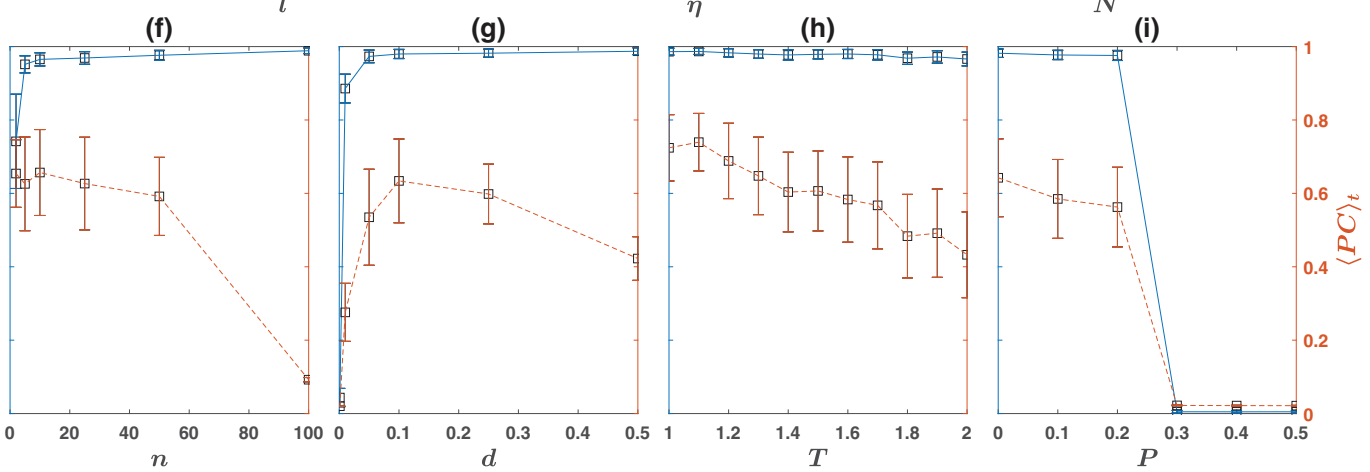

FIG. 7. Dependence on the model parameters in the model with unconditional cooperators on a first-nearest-neighbor square lattice. The time average cooperation level, $\langle m\rangle_{t}$ (blue), and the population consistency of the language (red), $\langle P C\rangle_{t}$, as a function of model parameters. Cooperation and a common language evolve and are maintained in a broad range of parameter values. $l_{t}$ is the number of correct communications necessary for a cooperator to play the game, $l$ is the number of coordination before a prisoner's dilemma game, $\eta$ is the amount of noise in parental learning. $N$ is the population size. $v$ is the mutation rate for the strategies, $n$ number of representations (equal to the number of signals), $d$ is the learning rate, $T$ the temptation, and $P$ the punishment. The simulations are performed for $10^{5}$ time steps and time averages and standard deviations (error bars) are calculated after discarding the first $10^{4}$ time steps.

stringent in playing the game. As here, the population starts with a random language, if the individuals are too strict, then the formation of a common language can be hindered. The reason is that, starting from a random language, the formation of a common language necessitates cooperators occasionally play the game with enough frequency. A high value of $l_{t}$, although can guard cooperators against being defected, hinders the formation of a common language as well. This in turn decreases the level of cooperation. A too small $l_{t}$ can be harmful as well. As such cooperators who easily cooperate, can play with defectors more often. This in turn, in addition to increasing the fitness of defectors and decreasing $m$, causes the cooperators who are defected change their language more often, which decreases $P C$.

The dependence on the strength of communication is investigated in Fig. 7(b). Here, $l_{t}$ is set to 1 and $\langle m\rangle_{t}$ and $\langle P C\rangle_{t}$ as a function of $l$ are plotted. As can be seen, even one communication instance $(l=1)$ can be enough for the emergence of cooperation. Furthermore, as $l$ increases, individuals have more chances to coordinate and both $P C$ and $m$ increase. However, for larger $l s$ the level of cooperation slightly decreases. The reason is that, with a fixed $l_{t}$, the probability that a conditional cooperator cooperates with a defector increases with increasing $l$. Such interactions favor defectors and thus, $m$ decreases. In addition, such interactions cause cooperators to constantly change their language as they are defected frequently. This in turn decreases $P C$.
Dependence on the amount of noise in parental learning, $\eta$, is investigated in Fig. 7(c). As can be seen, $P C$ decreases with increasing the amount of noise in parental learning. This seems intuitive as with a higher amount of noise in parental learning, offspring need to learn the language by their own. Nevertheless, the level of cooperation remains very high for all values of $\eta$. Interestingly, the level of cooperation slightly increases with increasing the amount of noise in parental learning. A similar phenomenon was observed in the model with conditional defectors and might seem to go against intuition, as one might expect increasing parental learning helps cooperators to preserve their language over time, and thus facilitates cooperation. The reason why increasing parental learning can slightly reduce cooperation level is that, a very large parental learning allows the defectors to learn the language by learning from cooperators' offspring in their neighborhood. This cannot happen for smaller parental learning, as in this case offspring's language bears less similarity with that of its parent and cannot be exploited by defectors as a door to the language.

The dependence on the population size $N$ is investigated in Fig. 7(d). As can be seen, the level of cooperation rapidly increases for very small populations and shows very little sensitivity to the population size, for large population sizes. $P C$ increases as well (much less rapidly) for small populations. For very small populations $(N<16)$ all the individuals live in close vicinity of each other. This gives defectors a good opportunity to learn the language of cooperators. As the 
population size increases, islands of cooperators are formed that live a considerable distance from defectors. Such small community of cooperators provide safe places for cooperators to coordinate their language and plays an important role in the formation and maintenance of cooperator's language. This is why $P C$ increases with population size, in small population sizes.

In Fig. 7(e), $\langle m\rangle_{t}$ and $\langle P C\rangle_{t}$ as a function of the mutation rate $v$ are plotted. As can be seen, both $\langle m\rangle_{t}$ and $\langle P C\rangle_{t}$ decrease with increasing mutation rate. The reason is easy to see, as with a higher mutation rate, cooperator's offspring with a high knowledge of cooperator's language turn defector too often. Having a high knowledge of cooperator's language, they can exploit cooperators very well. Cooperators in turn, being defected too often, constantly change their language to keep it incomprehensible for defectors. This, in turn, leads to a decrease in $P C$.

In Fig. 7(f) the dependence on the number of representation (equal to the number of signals) is investigated. As can be seen, while the level of cooperation slightly increases, $\langle P C\rangle_{t}$ decrease with increasing $n$. The reason is that, with fixed $l$ and $l_{t}$, by increasing $n$ the probability of correct communication decreases. Consequently, the number of mutual cooperation, which is necessary for building a common language, decreases. This reduces $P C$. Although detrimental for building a common language, increasing $n$ increases the level of cooperation. As decreasing the number of games played by the cooperators also decreases the opportunity of defectors to exploit them.

Dependence on the learning rate $d$ is investigated in Fig. 7(g). The level of cooperation increases with increasing $d$ for small $d$, and shows little sensitivity for larger $d$. The situation is different for $P C$. A very small $d$ leads to slow learning which lowers the ability of individuals to grow a common language. A large $d$, however, leads to too strong responses which is detrimental for coordination. A medium response is optimal for the evolution of a common language.

Finally, the dependence on temptation $T$ and punishment $P$ are investigated in Figs. 7(h) and 7(i). Cooperation remains large for all values of $T$ in the prisoner's dilemma range and slightly decreases with $T . P C$ shows stronger sensitivity and decreases with $T$ as well. By increasing the punishment, a sharp transition occurs to a regime where cooperation cannot be maintained. The reason is that defectors play the PD with each other and gather payoff $P$ from such interactions, even when they do not understand each other. In contrast, cooperators refrain from the game in cases when they do not understand their opponent, which leads to zero payoff which is smaller than the payoff of mutual defection. When a population starts with random languages such that the number of successful communication is low, cooperators refrain from too many games. This leads to a situation where defectors receive a higher payoff. Consequently, for large enough $P$ defection prevails in the population.
[1] R. Axelrod and W. D. Hamilton, The evolution of cooperation, Science 211, 1390 (1981).

[2] W. D. Hamilton, The genetical evolution of social behavior. II, J. Theor. Biol. 7, 17 (1964).

[3] S. A. West, A. S. Griffin, A. Gardner, and S. P. Diggle, Social evolution theory for microorganisms, Nature Rev. Microbiol. 4, 597 (2006).

[4] T. Clutton-Brock, Cooperation between nonkin in animal societies, Nature 462, 51 (2009).

[5] D. G. Rand and M. A. Nowak, Human cooperation, Trends Cogn. Sci. 17, 413 (2013).

[6] M. A. Nowak, Five rules for the evolution of cooperation, Science 314, 1560 (2006).

[7] M. Doebeli and C. Hauert, Models of cooperation based on the Prisoner's Dilemma and the Snowdrift game, Ecol. Lett. 8, 748 (2005).

[8] D. S. Wilson, A theory of group selection, Proc. Natl. Acad. Sci. USA 72, 143 (1975).

[9] A. Traulsen and M. A. Nowak, Evolution of cooperation by multilevel selection, Proc. Natl. Acad. Sci. USA 103, 10952 (2006).

[10] R. L. Trivers, The evolution of reciprocal altruism, Quart. Rev. Biol. 46, 35 (1971).

[11] M. A. Nowak and K. Sigmund, Evolution of indirect reciprocity by image scoring, Nature 393, 573 (1998).

[12] K. Panchanathan and R. Boyd, Indirect reciprocity can stabilize cooperation without the second-order free rider problem, Nature 432, 499 (2004).

[13] M. A. Nowak and K. Sigmund, Evolution of indirect reciprocity, Nature 437, 1291 (2005).
[14] M. A. Nowak and R. M. May, Evolutionary games and spatial chaos, Nature 359, 826 (1992).

[15] H. Ohtsuki, C. Hauert, E. Lieberman, and M. A. Nowak, A simple rule for the evolution of cooperation on graphs and social networks, Nature 441, 502 (2006).

[16] R. L. Riolo, M. D. Cohen, and R. Axelrod, Evolution of cooperation without reciprocity, Nature 414, 441 (2001).

[17] V. A. Jansen and M. Van Baalen, Altruism through beard chromodynamics, Nature 440, 663 (2006).

[18] C. Hauert, S. De Monte, J. Hofbauer, and K. Sigmund, Volunteering as red queen mechanism for cooperation in public goods games, Science 296, 1129 (2002).

[19] G. Szabó and C. Hauert, Phase Transitions and Volunteering in Spatial Public Goods Games, Phys. Rev. Lett. 89, 118101 (2002).

[20] E. Fehr and S. Gächter, Altruistic punishment in humans, Nature 415, 137 (2002).

[21] M. Perc et al., Statistical physics of human cooperation, Phys. Rep. 687, 1 (2017).

[22] R. Boyd, H. Gintis, and S. Bowles, Coordinated punishment of defectors sustains cooperation and can proliferate when rare, Science 328, 617 (2010).

[23] A. Szolnoki and M. Perc, Second-Order Free-Riding on Antisocial Punishment Restores the Effectiveness of Prosocial Punishment, Phys. Rev. X 7, 041027 (2017).

[24] A. L. Bazzan and S. R. Dahmen, Bribe and punishment: Effects of signaling, gossiping, and bribery in public goods games, Adv. Complex Syst. 13, 755 (2010). 
[25] A. Szolnoki and M. Perc, Antisocial pool rewarding does not deter public cooperation, Proc. R. Soc. B 282, 20151975 (2015).

[26] C. Hilbe and K. Sigmund, Incentives and opportunism: From the carrot to the stick, Proc. R. Soc. B 277, 2427 (2010).

[27] M. Salahshour, Evolution of costly signaling and partial cooperation, Sci. Rep. 9, 8792 (2019).

[28] Eric Alden Smith, Communication and collective action: Language and the evolution of human cooperation, Evol. Hum. Behav. 31, 231 (2010).

[29] Edited by D. K. Oller and U. Griebel, 13: Cooperation and the Evolution of Symbolic Communication, in Evolution of Communication Systems: A Comparative Approach (MIT Press Direct, 2004).

[30] P. Gärdenfors, 8. The Role of Cooperation in the Evolution of Protolanguage and Language, in Evolution of Mind, Brain, and Culture, edited by G. Hatfield and H. Pittman (University of Pennsylvania Press, Pennsylvania, 2013), pp. 193-216.

[31] J. Ke, J. W. Minett, C. P. Au, and W. S. Y. Wang, Selforganization and selection in the emergence of vocabulary, Complexity 7, 41 (2002).

[32] M. A. Nowak, and N. L. Komarova, Towards an evolutionary theory of language, Trends Cognit. Sci. 5, 288 (2001).

[33] M. A. Nowak, D. C. Krakauer, and A. Dress, An error limit for the evolution of language, Proc. R. Soc. London B 266, 2131 (1999).

[34] M. A. Nowak and D. C. Krakauer, The evolution of language, Proc. Natl. Acad. Sci. USA 96, 8028 (1999).
[35] J. R. Hurford, Biological evolution of the Saussurean sign as a component of the language acquisition device, Lingua 77, 187 (1989).

[36] G. Jäger, Applications of game theory in linguistics, Lang. Ling. Compass 2, 406 (2008).

[37] M. Salahshour, S. Rouhani, and Y. Roudi, Phase transitions and asymmetry between signal comprehension and production in biological communication, Sci. Rep. 9, 3428 (2019).

[38] M. Salahshour, Phase Diagram and Optimal Information Use in a Collective Sensing System, Phys. Rev. Lett. 123, 068101 (2019).

[39] L. C. Aiello and R. I. Dunbar, Neocortex size, group size, and the evolution of language, Curr. Anthropol. 34, 184 (1993).

[40] G. Szabó and G. Fath, Evolutionary games on graphs, Phys. Rep. 446, 97 (2007).

[41] V. Loreto, A. Baronchelli, A. Mukherjee, A. Puglisi, and F. Tria, Statistical physics of language dynamics, J. Stat. Mech. (2011) P04006.

[42] A. Baronchelli, M. Felici, V. Loreto, E. Caglioti, and L. Steels, Sharp transition towards shared vocabularies in multi-agent systems, J. Stat. Mech. (2006) P06014.

[43] S. Gächter and C. Thöni, Social learning and voluntary cooperation among like-minded people, J. Eur. Econ. Assoc. 3, 303 (2005).

[44] D. Balliet, J. Wu, and C. K. De Dreu, Ingroup favoritism in cooperation: A meta-analysis, Psychol. Bull. 140, 1556 (2014). 